\title{
Neural Oscillations in Antipsychotic-Naïve Patients with a First Psychotic Episode
}

\author{
Avinash Ramyead ${ }^{1}, \mathrm{MSc}$; Erich Studerus ${ }^{1}, \mathrm{PhD}$; Michael Kometer ${ }^{2}, \mathrm{PhD}$; Ulrike Heitz ${ }^{1}$, \\ MSc; Ute Gschwandtner ${ }^{3}$, MD; Peter Fuhr ${ }^{3}$, MD; Anita Riecher-Rössler ${ }^{1^{*}}, M D$ \\ ${ }^{1}$ University of Basel Psychiatric Clinics, Center for Gender Research and Early Detection, Basel, Switzerland \\ ${ }^{2}$ Neuropsychopharmacology and Brain Imaging Research Unit, Department of Psychiatry, Psychotherapy and \\ Psychosomatics, Hospital of Psychiatry, University of Zurich, Switzerland \\ ${ }^{3}$ Department of Neurology, University Hospital Basel, Basel, Switzerland
}

Running title: Neural Oscillations in Emerging Psychosis

\section{*Corresponding Author:}

Prof. Anita Riecher-Rössler, MD

University of Basel Psychiatric Clinics

Center for Gender Research and Early Recognition

Kornhausgasse 7

CH-4051 Basel, Switzerland

Phone: +41613258161

Fax: +41613258160

E-mail: anita.riecher@upkbs.ch

Number of words in article body: 4321

Number of figures: 4

Number of tables: 1 


\begin{abstract}
Objectives: In chronic schizophrenic psychoses, oscillatory abnormalities predominantly occur in prefrontal cortical regions and are associated with reduced communication across cortical areas. Nevertheless, it remains unclear whether similar alterations can be observed in patients with a first-episode of psychosis (FEP), a state characterized by pathological features occurring in both late prodromal patients and initial phases of frank schizophrenic psychoses.
\end{abstract}

Methods: We assessed resting-state EEG data of 31 antipsychotic-naïve FEP patients and 29 healthy-controls (HC). We investigated the 3-dimensional current-source density (CSD) distribution and lagged phase synchronization (LPS) of oscillations across small-scale and largescale brain networks. We additionally investigated LPS relationships with clinical symptoms using linear mixed-effects models.

Results: Compared to HC, FEP patients demonstrated abnormal CSD distributions in frontal areas of the brain; while decreased oscillations were found in the low frequencies, an increase was reported in the high frequencies $(p<0.01)$. Patients also exhibited deviant LPS in the high frequencies, whose dynamics changed over increasing 3D cortico-cortical distances and increasing psychotic symptoms.

Conclusions: These results indicate that in addition to prefrontal cortical abnormalities, altered synchronized neural oscillations are also present, suggesting possible disruptions in corticocortical communications. These findings provide new insights into the pathophysiological mechanisms of emerging schizophrenic psychoses.

Key words: Schizophrenia, Psychosis, EEG, Phase synchrony, Biomarkers 


\section{Introduction}

Various studies in patients with schizophrenia have investigated the hypofrontality and hyperfrontality hypotheses, which state that these patients have an inability to activate or deactivate frontal regions of the brain (Guerrero-Pedraza et al. 2012). While some findings on patients with first-episode schizophrenia support the hyperfrontality hypothesis (Schneider et al. 2007; Whitfield-Gabrieli et al. 2009; Woodward et al. 2009), other studies observed a hypofrontality (Nejad et al. 2011; Tan et al. 2005). However, most of these studies have made use of fMRI or PET, which do not measure brain activity at different frequency bands, which are known to have distinct dynamical properties, particularly in the schizophrenic brain (Uhlhaas et al. 2010).

Schizophrenic psychoses are now acknowledged as neurodevelopmental disorders, whose initial signs and symptoms can sometimes be observed as early as in childhood (Insel 2010). Compared to first-episode schizophrenia, a first-episode of psychosis (FEP) is a state characterized by pathological features occurring in both late prodromal patients and initial phases of frank schizophrenic psychoses (Sumiyoshi et al. 2008), but do not necessarily fulfill the diagnosis criteria of schizophrenia. Therefore, studies investigating brain abnormalities in FEP patients are clearly needed to bridge the gap between the numerous investigations on patients at-risk for psychosis and those already diagnosed with schizophrenia. In particular, investigations of neural oscillations in FEP patients are rare and those investigating antipsychotics-naïve populations are even scarcer. This is unfortunate as some antipsychotics have been shown to alter low frequency oscillations, particularly in the alpha band (Centorrino et al. 2014; Kikuchi et al. 2007). Moreover, the few studies on FEP patients assessing resting state EEG lacked a control group (Gschwandtner et al. 2009), or have assessed the EEG only qualitatively (Manchanda et al. 2003; Manchanda et al. 2008; Manchanda et al. 2014; Manchanda et al. 2005). Other studies used auditory paradigms and demonstrated increased gamma synchronization and reduced global field power in FEP 
patients (Flynn et al. 2008; Valkonen-Korhonen et al. 2003). Thus, in the present study, we investigated a relatively large and rare sample of FEP patients, who had not yet been treated by antipsychotics at time of their clinical EEG session. These patients were recruited by our early detection center for psychosis, $\boldsymbol{F e P s y}$, which has been specially designed to detect and treat emerging psychosis at its very early stages (Riecher-Rössler et al. 2007). A crucial aim of the current study was to elucidate whether this particular group of patients demonstrates alterations in low and high frequency oscillations. We further aimed to reveal the spatial distribution of these neural oscillations by quantifying reference-free, three-dimensional current source density (CSD) across brain areas. CSD has been shown to be robust against volume conduction while sharpening its spatial resolution (Nunez et al. 2006).

In addition to an alteration in CSD of neural oscillations, a disruption of phase-synchronization of neural oscillations across brain areas may also be a crucial characteristic of FEP patients. This is in line with the disconnection hypothesis (Buchsbaum et al. 2014; Schmitt et al. 2011), which states that in the schizophrenic brain, cortical regions fail to communicate and synchronize themselves. As these cortico-cortical communications are modulated primarily through phase synchronization (Fell et al. 2011; Wang et al. 2011), which was found to be impaired in the beta and gamma bands in schizophrenia (Uhlhaas et al. 2010; 2013), we investigated whether phase synchronization between distributed brain regions is abnormal at different frequency bands in patients with a first psychotic episode. Finally, because phase synchronization plays a crucial role in various cognitive processes (Doesburg et al. 2008; Ward 2003), we tested the idea that deviant phase synchronizations across cortical areas are associated with positive and/or negative symptoms. To address these questions, we employed a new and non-linear measure of brain connectivity, namely lagged phase synchronization (LPS), which has been shown to be minimally affected by volume conduction and low spatial resolution (due to the extraction of zero-lag LPS), 
thus retaining most of the true neurophysiological connectivity (Pascual-Marqui 2007; PascualMarqui et al. 2011).

We hypothesized that in FEP patients, a decrease in oscillatory activity would be observed in some frequencies while an increase would be observed in others. Moreover, we also hypothesized that the LPS across cortical regions would be altered in FEP particularly in frequencies that have been shown to be involved in long-range synchronization (i.e. theta, alpha, beta and not gamma (Uhlhaas et al. 2010)) and that positive and negative symptoms would be associated with these abnormalities.

\section{Methods}

\section{Setting and Recruitment}

Patients recruited for this study were help-seeking consecutive referrals to the $\boldsymbol{F e P s y}$ Clinic at the University Psychiatric Clinics Basel, which was set up to assess, measure, and treat individuals in the early stages of psychosis (Riecher-Rössler et al. 2009; Riecher-Rössler et al. 2007). This study was approved by the ethics committee of the University of Basel, and all participants provided written informed consent.

\section{Screening Procedure}

The Basel Screening Instrument for Psychosis (BSIP, Riecher-Rössler et al. 2008), was used to identify FEP patients. The BSIP allows the rating of individuals regarding the inclusion/exclusion criteria according to the PACE criteria (Yung et al. 1998) and has been shown to have a high predictive validity and a good interrater reliability (Riecher-Rössler et al. 2008). Exclusion criteria for patients includes: age $<18$ years, inadequate knowledge of German, IQ $<70$ as measured by the Mehrfachwahl-Wortschatz (Test Form A), previous episode of schizophrenic psychosis treated with antipsychotics, psychosis clearly due to organic reasons or substance abuse, or psychotic symptoms within a clearly diagnosed depression or borderline personality disorder. We 
included FEP patients that were recruited between March 2000 and January 2013 and had a clinical EEG session of at least 15 minutes at baseline assessment.

The FEP patients met the criteria for having transitioned to schizophrenic psychosis according to Yung and colleagues (Yung et al. 1998). While these patients fulfilled criteria for acute psychotic disorder according to the DSM-IV or ICD-10, they did not necessarily yet meet the criteria of schizophrenia. All FEP patients were antipsychotic and mood-stabilizer naive at time of EEG assessment.

Healthy controls (HC) were recruited from the same geographical area as the patients, through advertisements in trade schools and from the hospital staff. Exclusion criteria for the healthy participants were: history of psychiatric or neurological disease, past or present substance abuse and head trauma.

\section{Assessment of Positive and Negative Psychotic Symptoms}

The Brief Psychiatric Rating Scale Expanded (BPRS-E, Lukoff et al. 1986; Ventura et al. 1993) was used to assess positive and negative psychotic symptoms. The positive psychotic symptom scale was based on the four items hallucinations, suspiciousness, unusual thought content, and conceptual disorganization and the negative psychotic symptom scale was based on the items blunted affect, psychomotor retardation and emotional withdrawal (Velligan et al. 2005).

\section{EEG Recordings}

EEG data were recorded at the University Hospital of Basel. Patients sat in a quiet room during eyes closed resting-state condition for about 20 minutes. Every three minutes and/or at signs of behavioral and/or EEG drowsiness, subjects were asked to open their eyes for a period of 5-6 seconds. EEG data were sampled at $250 \mathrm{~Hz}$ by 19 gold cup electrodes (Nicolet Biomedical Inc), referenced to linked ears and impedances kept below $5 \Omega$. 


\section{Artifact Rejection}

EEG pre-processing was performed using Brain Vision Analyzer@ 2.0 software (Brain Products $\mathrm{GmbH})$. We processed each EEG in parallel split into two branches, one filtered at $0.5 \mathrm{~Hz}$ and one at $1 \mathrm{~Hz}$. We did so in order to apply the ICA matrix from the most stable signal $(1 \mathrm{~Hz})$ to the one that conserved the most signal $(0.5 \mathrm{~Hz})$. Both branches were handled in the same way up to the step that involved re-referencing to the common average. Artifact rejection was performed manually, based on visual inspection, to remove epochs containing extreme ocular artifacts, muscles and/or cardiac contamination and bad signals due to random movements. Biased extended Infomax ICA analyses were then performed for the removal of residual eye movements, eye-blinking, muscles and non-biological components contaminated with high gamma frequencies of $50 \mathrm{~Hz}$ and above as measured by Fast Fourier Transform (FFT) of the ICA components (resolution at $1 \mathrm{~Hz}$, power $\mu \mathrm{V}^{2}$, hanning window length of $10 \%$ ). After applying the ICA corrected matrix of the data filtered at $1 \mathrm{~Hz}$ to the one filtered at $0.5 \mathrm{~Hz}$, data were re-referenced to common average. A final manual rejection based on visual inspection was performed to exclude remaining artifacts as mentioned above

\section{CSD Analyses}

The EEG electrode montage in the present study is in accordance with previous recent studies assessing patients (Babiloni et al. 2013; Canuet et al. 2011b; Canuet et al. 2012; Ramyead et al. 2014) and is considered to allow adequate EEG spatial sampling for the estimation of cortical sources of eyes-closed resting-state EEG rhythms (Babiloni et al. 2013). Accordingly, the oscillatory rhythms acquired during eye-closed resting-state EEG can be sampled with a relatively low number of electrodes, as opposed to the higher density electrode montage required for observing the functional topography of stimuli-related EEG activity (Babiloni et al. 2013). Computing the intracortical CSD of oscillatory activity was performed using eLORETA (PascualMarqui 2007; Pascual-Marqui et al. 2011). This was based on EEG data segmented into 2s epochs 
(638 2s epochs on average, groups did not differ in number of epochs). The cross-spectra were computed as the average of all cross spectra of each individual EEG epoch.

As opposed to conventional EEG analyses based on voltage, the use of 3D CSD as a measure of brain activity allows for a reliable spatial analysis (Michel et al. 2004) by disentangling the EEG signals from various biological and non-biological artifacts, therefore yielding measures more faithfully representing the neuronal current generators (Tenke et al. 2011). The neurophysiological imaging technique eLORETA is based on a weighted minimum norm inverse solution procedure which allows for the 3D modeling of the EEG CSD with an exact localization performance, with a high correlation of neural sources that are in close proximity. LORETA has been validated as an efficient and reliable tool to study brain activity by various multi-modal studies. These include neuroimaging studies such as functional (Mulert et al. 2004) and structural MRI (Worrell et al. 2000), PET (Pizzagalli et al. 2003; Zumsteg et al. 2005) and intracranial EEG recordings (Zumsteg et al. 2006). As opposed to the first version of LORETA (Pascual-Marqui et al. 1994), the third iteration eLORETA has no localization bias in the presence of structured noise (Pascual-Marqui 2007).

eLORETA assumes a head model based on 3 shells (brain, scalp and skull compartments) and the solution space is restricted to the cortical grey matter/hippocampus, which comprises 6239 voxels of $5 \mathrm{~mm}^{3}$ each. Computing the lead field in the above-mentioned head model is based on the Montreal Neurological Institute brain MRI average (Mazziotta et al. 2001). CSD analyses were based on the following frequency bands: delta $(1.5-4 \mathrm{~Hz})$, theta $(4-8 \mathrm{~Hz})$, alpha1 $(8-10 \mathrm{~Hz})$, alpha2 $(10-13 \mathrm{~Hz})$, beta1 $(13-21 \mathrm{~Hz})$, beta2 $(21-30 \mathrm{~Hz})$ and gamma $(30-50 \mathrm{~Hz})$.

\section{LPS Analyses}

To compute the phase synchronization, we defined 19 regions of interests (ROIs) spread along the cortex (Canuet et al. 2012; Ramyead et al. 2014). These ROIs were based on the Montreal Neurological Institute (MNI) coordinates of the cortical voxel (Table S1 and more LPS technical 
details in supplementary appendix 1). Activity at centroid voxels for each ROI was extracted. We then computed the LPS between all the 19 ROIs resulting in 171 pairwise combinations. LPS computes the non-linear relationship between each pair after the instantaneous zero-lag contribution has been removed. This results in the elimination of non-physiological artifacts such as volume conduction (Pascual-Marqui et al. 2011). To assess the phase synchronization in relation to distance, we calculated the Euclidian distance between the first ROI (x1, y1, z1) and the second $(x 2, y 2, z 2)$ using the Pythagorean theorem: $\sqrt{ }\left[(x 2-x 1)^{2}+(y 2-y 1)^{2}+(z 2-z 1)^{2}\right]$ which were then standardized into Z-scores.

\section{Statistical Analyses}

In order to identify the CSD differences between FEP and $\mathrm{HC}$, we used the statistical nonparametric mapping (SnPM) implemented in eLORETA (Holmes et al. 1996), which has been validated (Anderer et al. 1998; Pascual-Marqui et al. 1999) and used in previous clinical studies (Canuet et al. 2011a; Canuet et al. 2012; Ramyead et al. 2014). Differences in cortical oscillations through each frequency band were calculated by voxel-by-voxel independent sample t-statistics with electrode/voxel-wise normalization (relative power type). Subsequently, 5000 permutations were used to perform randomized SnPM and correct for the critical probability threshold across all voxels and all frequencies (1\% probability level).

Due to the age difference between the HC and FEP groups (Table 1), we assessed whether age was associated with the deviant oscillatory activity revealed using the methods above. Thus, we extracted CSD values from the global maximum voxel at corresponding frequencies that differed between FEP and HC. Afterwards, their association was assessed by linear regression models using CSD as dependent variables and centered age and diagnostic group as independent variables. In addition to this ROI approach, a brain-wide analysis was performed by correlating voxel-wise age with CSD within eLORETA for each frequency. This whole brain analysis was 
based on 5000 permutations to determine the empirical probability distribution for the maximal statistics under the null hypothesis (Canuet et al. 2012; Hubl et al. 2007).

To assess group differences in LPS, we fitted a linear mixed-effects model using LPS values from 171 pairs as the dependent variable and the centered Euclidian distance (within-subjects) and group (between-subjects) along with their interaction as independent variables. Moreover, the model included an intercept term that randomly varied per individual. To control for heteroscedasticity, we explicitly modeled the variance in the model by adding a constant plus power variance function structure. To examine the association between positive/negative symptoms and lagged phase synchronization as a function of Euclidian distances, we fitted linear mixed-effects models that additionally included the centered BPRS positive and BPRS negative symptom scores as fixed effects. These analyses were repeated for each of the seven different frequencies and were controlled for false discovery rates using the Benjamini-Hochberg method (Benjamini et al. 1995).

\section{Results}

\section{Sample Description}

From February 2000 to January 2013, 99 FEP patients and 97 HC have been recruited into the FePsy study. Of these, only 31 FEP patients and $29 \mathrm{HC}$ had sufficient (at least 15 minutes) clinical EEG data and were antipsychotic and mood-stabilizer naive. Four of these patients were currently on antidepressants and 8 were on tranquilizers. The 68 FEP individuals that could not be included due to not having had an EEG session and/or were already medicated with antipsychotics did not differ from the included FEP individuals with regard to gender, age, years of education, positive and negative BPRS total, positive symptoms scores. The clinical characteristics and demographics of the HC and FEP groups are shown in Table 1. There was a difference in age and a small difference in years of education (all $p$ 's $<.05)$. 


\section{Source Localization}

For illustrative purpose, CSD distributions for each group at each frequency band are present in Figure 1. Non-statistically, in HC, highest oscillatory activities were found in the alpha2 band ( 0.55 vs. $0.30 \mu \mathrm{A} / \mathrm{mm}^{2}$ in $\mathrm{HC}$ and FEP, respectively) followed by the delta band (0.43 vs. $0.33 \mu \mathrm{A} / \mathrm{mm}^{2}$ ). In contrast, in FEP patients the highest oscillatory activities were in the gamma (0.87 vs. $0.37 \mu \mathrm{A} / \mathrm{mm}^{2}$ in FEP and $\mathrm{HC}$, respectively) and alpha1 $\left(0.38\right.$ vs. $\left.0.38 \mu \mathrm{A} / \mathrm{mm}^{2}\right)$ bands. Regarding the spatial distribution of CSD, statistical analyses revealed that FEP patients had decreased theta activity in the left anterior cingulate (BA32, global maximum at $\mathrm{X}=-15, \mathrm{Y}=35$, $\mathrm{Z}=20, \mathrm{t}=-4.40, p^{<.01}$, corrected) and decreased alpha1 activity in the left middle frontal gyrus (BA10, global maximum at $\mathrm{X}=-30, \mathrm{Y}=60, \mathrm{Z}=10, \mathrm{t}=-4.05, p<.01$, corrected). Moreover, FEP patients also had increased activity in the beta2 band bilaterally in the superior frontal gyrus, particularly in the left hemisphere (BA8, global maximum at $\mathrm{X}=-20, \mathrm{Y}=30, \mathrm{Z}=55, \mathrm{t}=4.23, p<.01$, corrected), and increased gamma activity in the left medial frontal gyrus (BA9, global maximum at $\mathrm{X}=-20, \mathrm{Y}=35, \mathrm{Z}=25, \mathrm{t}=3.75, p<.01$, corrected).

\section{CSD and Age}

Four Linear regression models with CSD activity, individually extracted at the global maximum voxel from the theta, alpha1, beta2 and gamma frequency bands, as dependent variable and age and group as independent variables revealed no significant main effect of age (all $4 p$ 's $>0.80$, corrected). A whole brain voxel-wise correlations analysis also demonstrated that age was not associated with CSD measurements for any frequencies ( $p>.20$, corrected).

\section{LPS Analyses}

Linear mixed-effects models with LPS values as dependent variables, Euclidian distance, group and their interaction as independent variable and a random intercept per subject revealed significant main effects of Euclidian distance for all frequency bands $(p<0.05$ for the delta frequency and $p<0.001$ for all 6 remaining frequencies, corrected). This was due to decreased LPS with increasing distances between the ROIs (171 pairs) in all frequencies except for the delta band 
(Figure S1A, supplementary appendix 1). Moreover, there was a significant interaction between group and Euclidian distance for LPS of beta1 and beta2 oscillations $(p<.05$ and $p<0.001$, respectively, corrected for 7 comparisons), which was due to a stronger decrease of LPS with increasing anatomical distance in FEP patients than in HC (Figure 3). In the delta and alpha1 frequency bands similar interactions were observed, which however were not significant, possibly due to rigorous correction for multiple comparisons $(p<.10$, corrected, Figure S1A, supplementary appendix 1).

In the linear mixed-effect models that also included BPRS positive symptoms as an independent variable, a significant second-order interaction between LPS, distance and BPRS positive symptoms in the beta2 frequency bands was revealed ( $p<0.001$, corrected), indicating that higher positive symptoms in FEP patients were associated with a particularly strong decrease of LPS with increasing distance, which was exaggerated with increasing positive symptoms (Figure 4A). Furthermore, the model with negative symptoms revealed a main effect of BPRS negative symptoms on a trend level ( $p=0.05$, corrected) and a second order interaction between LPS, distance and BPRS negative solely in the beta1 band ( $p<0.001$, corrected, Figure 4$)$.

\section{Discussion}

In this study we investigated whether antipsychotic-naïve FEP patients demonstrated deviant CSD and LPS when compared to healthy individuals. We found decreased CSD of theta and alpha1 oscillations in the left frontal cortex, but increased beta2 CSD in fronto-parietal areas and increased gamma oscillations in the left frontal cortex. We additionally found an inverse relationship between LPS and Euclidian distance in the beta1 and beta2 bands, which was stronger in FEP compared to HC individual for betal and less strong in FEP compared to HC individual for beta2. 


\section{CSD Analyses}

This study emphasizes and demonstrates that both a hypofrontality and a hyperfrontality are concurrently present in emerging schizophrenic psychosis. While a hypofrontality in CSD is observed in the low theta and alphal frequencies, a hyperfrontality has been revealed in high beta 2 and gamma bands. The mid frequencies such as alpha2 and beta1 were not associated with localized abnormal oscillatory activity. These results extend previous studies focusing on cerebral blood flow during rest (Guerrero-Pedraza et al. 2012; Whitfield-Gabrieli et al. 2009) that could not assess activity at different frequency bands.

The revealed abnormality in the theta band specifically in the left anterior cingulate cortex (1ACC) is in line with a study, which has revealed that unmedicated patients with a first episode of schizophrenia have higher than normal glutamine levels in the 1ACC resulting in reduced glutamatergic activity (Kegeles et al. 2012; Théberge et al. 2002). Deregulations at this location has been shown to alter theta oscillatory activity and working memory (Holscher et al. 2005). Moreover, ACC theta oscillations in non-human primates have been shown to predict task rules comprehension, adjustments to errors (Womelsdorf et al. 2010) and various other attentional processes (Tsujimoto et al. 2006), all of which have been shown to be impaired in schizophrenia (Mesholam-Gately et al. 2009).

Although both the HC and the FEP groups had the same cortical average CSD in the alpha1 frequency band (Figure 1), FEP patients had significantly lower CSD in the left middle frontal gyrus. Only few studies have reliably revealed deviant frontal alpha oscillations in schizophrenia (Knyazeva et al. 2008). One potential explanation could be that we investigated the alpha band (8$13 \mathrm{~Hz})$ split into two more refined frequency bands namely alpha1 $(8-10 \mathrm{~Hz})$ and alpha2 (10$13 \mathrm{~Hz}$ ), which have been shown in healthy human subjects to have different dynamic properties (Knyazev et al. 2003; Micheloyannis et al. 2006; Mu et al. 2008). Moreover, antipsychotics have been found to normalize oscillations, particularly alpha oscillations (Centorrino et al. 2014; 
Kikuchi et al. 2007), possibly by their antagonistic activity at 5-HT2A receptors (Kometer et al. 2013), which may explain why we revealed decreased alpha oscillations compared to previous studies assessing antipsychotic-treated schizophrenic patients.

The increased CSD in the beta2 bands on both hemispheres, that is, across both superior frontal gyri, could be due to oligodendrocytes loss that has been reported in patients with schizophrenia in this area (Hof et al. 2003) which play an important role in promoting neural synchrony (Fields 2008; Schmitt et al. 2011). Furthermore, oligodendrocytes contain NMDA receptors (Káradóttir et al. 2005), thus, a reduction would also result in reduced NMDA receptor activations and consequently reduced GABAergic inhibition (Koch et al. 2015). Furthermore, this process has been suggested to increase beta2 oscillatory activity and cortical gamma rhythms (Koch et al. 2015; Roopun et al. 2008). In accordance with this, the present study also revealed an increase in gamma oscillations in frontal regions.

The increased frontal gamma oscillations in FEP patients is in accordance with several previous studies on schizophrenic psychoses (Hirano et al. 2015; Uhlhaas et al. 2013). Even though both an increase and a decrease of gamma oscillatory activity have been observed in patients suffering from psychosis, converging evidence suggests that an increase is mostly present in unmedicated patients exhibiting positive symptoms (Lee et al. 2003). In support of this, a recent study has shown an increase in resting-state frontal gamma activity already in patients at-risk for psychosis who later transitioned to psychosis but not in those who did not (Ramyead et al. 2014) and was found to be, along other predictors, predictive of transitions to psychosis (Ramyead et al. 2015).

Taken together, these findings support the notion that, in FEP, both hyper- and hypo-activations are present in frontal cortical areas. Alterations in the low frequencies have only been observed in few previous studies (Gschwandtner et al. 2009; Kim et al. 2015; Knyazeva et al. 2008). One possible reason for this discrepancy is that in the present study only antipsychotic-naïve patients were included, whereas most previous investigations had studied patients under the influence of 
antipsychotics, which might have obfuscated the detection of these alterations (Centorrino et al. 2014; Kikuchi et al. 2007).

\section{LPS Analyses}

Our results show that FEP patients demonstrate a stronger decrease in LPS with increasing Euclidian distance in the beta1 band (Figure 3A) than HC. Furthermore, an inverse association with LPS and Euclidian distance was increased with increasing positive symptoms (Figure 4A). Surprisingly, the opposite group $\mathrm{x}$ distance interaction was revealed in the beta2 band (Figure 3A) and was associated with negative symptoms (Figure 4B).

The heightened synchronization among cortical areas closest in proximity, i.e. at low Euclidian distances in relation to LPS values in the beta1 band, could reveal a perturbation of long-range synchronization in the psychotic brain. These findings could be due to altered anatomical connections in terms of cortical thickness and volume in schizophrenia (Oertel-Knöchel et al. 2013; Pol et al. 2014), which have been shown to alter beta oscillations (Uhlhaas et al. 2010), thus potentially leading to a poorer communication among numerous cortical areas. Furthermore, these results support the disconnectivity hypothesis, which has been described more than 40 years ago (Beaumont et al. 1973) and is amongst the best supported hypotheses today (Buchsbaum et al. 2014; Schmitt et al. 2011). Interestingly, our results are in line with a previous study, which revealed that at-risk patients who later developed schizophrenic psychosis had similar deviant LPS in the betal band compared to those without later transitions (Ramyead et al. 2014), thus further supporting that schizophrenic psychoses are indeed developmental disorders and that some deviances in oscillatory rhythms could be observed at a very early stage of the disease.

\section{Limitations}

A limitation of this study is that the EEG data was obtained with a relatively low density EEG equipment. Although some recent studies have used a similar system for resting-state sourcelocalization and connectivity measurements (Babiloni et al. 2013; Canuet et al. 2011b; Canuet et 
al. 2012; Ramyead et al. 2014), a higher density system would have yielded more precise results. Moreover, even though all patients were never medicated with antipsychotics and moodstabilizers, some of these patients were on antidepressants and tranquilizers, which could have had some influence over the results. Moreover, since it has been shown that bipolar patients are frequently misdiagnosed as FEP at initial presentation (Altamura et al. 2015), this could also have happened to some of our FEP patients.

\section{Conclusions}

Our findings reveal that both a hypofrontality and hyperfrontality are present in antipsychoticnaïve patients with a FEP, which are observed in the low and high frequencies, respectively. Moreover, the observed increased lagged phase synchronization across smaller inter-cortical areas in the betal frequency may well result in poor communications across the brain and could potentially arise from anatomical abnormalities.

\section{Acknowledgments}

This work was supported by the Swiss National Science Foundation (P0BSP1-152074, 3200057216.99, 3200-0572216.99, PBBSB-106936, 3232BO-119382). The authors would like to thank the patients and volunteers for participating in this study.

\section{Statement of Interest}

None to declare. 


\section{References}

Altamura AC, Buoli M, Caldiroli A, Caron L, Melter CC, Dobrea C, Cigliobianco M, Quarantini FZ. 2015. Misdiagnosis, duration of untreated illness (DUI) and outcome in bipolar patients with psychotic symptoms: A naturalistic study. Journal of affective disorders 182:70-75.

Anderer P, Pascual-Marqui RD, Semlitsch HV, Saletu B. 1998. Differential effects of normal aging on sources of standard N1, target N1 and target P300 auditory event-related brain potentials revealed by low resolution electromagnetic tomography (LORETA). Electroencephalography and Clinical Neurophysiology/Evoked Potentials Section 108(2):160-174.

Babiloni C, Carducci F, Lizio R, Vecchio F, Baglieri A, Bernardini S, Cavedo E, Bozzao A, Buttinelli C, Esposito F, Giubilei F, Guizzaro A, Marino S, Montella P, Quattrocchi CC, Redolfi A, Soricelli A, Tedeschi G, Ferri R, Rossi-Fedele G, Ursini F, Scrascia F, Vernieri F, Pedersen TJ, Hardemark HG, Rossini PM, Frisoni GB. 2013. Resting state cortical electroencephalographic rhythms are related to gray matter volume in subjects with mild cognitive impairment and Alzheimer's disease. Hum Brain Mapp 34(6):1427-46.

Beaumont J, Dimond S. 1973. Brain disconnection and schizophrenia. The British Journal of Psychiatry.

Benjamini Y, Hochberg Y. 1995. Controlling the false discovery rate: a practical and powerful approach to multiple testing. Journal of the Royal Statistical Society. Series B (Methodological):289-300.

Buchsbaum M, Christian BT, Merrill B, Lehrer DS. Disconnection of Striatum, Hippocampus, and Cortex Assessed with 18F-Fallypride PET Binding in Schizophrenia. NEUROPSYCHOPHARMACOLOGY; 2014: NATURE PUBLISHING GROUP MACMILLAN BUILDING, 4 CRINAN ST, LONDON N1 9XW, ENGLAND. p. S238-S238.

Canuet L, Ishii R, Iwase M, Ikezawa K, Kurimoto R, Takahashi H, Currais A, Azechi M, Aoki Y, Nakahachi T, Soriano S, Takeda M. 2011a. Psychopathology and working memory-induced activation of the prefrontal cortex in schizophrenia-like psychosis of epilepsy: Evidence from magnetoencephalography. Psychiatry Clin Neurosci 65(2):183-90.

Canuet L, Ishii R, Pascual-Marqui RD, Iwase M, Kurimoto R, Aoki Y, Ikeda S, Takahashi H, Nakahachi T, Takeda M. 2011b. Resting-state EEG source localization and functional connectivity in schizophrenia-like psychosis of epilepsy. PloS one 6(11):e27863.

Canuet L, Tellado I, Couceiro V, Fraile C, Fernandez-Novoa L, Ishii R, Takeda M, Cacabelos R. 2012. Resting-state network disruption and APOE genotype in Alzheimer's disease: a lagged functional connectivity study. PLoS One 7(9):e46289.

Centorrino F, Price BH, Tuttle M, Bahk W-M, Hennen J, Albert MJ, Baldessarini RJ. 2014. EEG abnormalities during treatment with typical and atypical antipsychotics.

Doesburg SM, Roggeveen AB, Kitajo K, Ward LM. 2008. Large-scale gamma-band phase synchronization and selective attention. Cerebral Cortex 18(2):386-396.

Fell J, Axmacher N. 2011. The role of phase synchronization in memory processes. Nature reviews neuroscience 12(2):105-118.

Fields RD. 2008. Oligodendrocytes changing the rules: action potentials in glia and oligodendrocytes controlling action potentials. The Neuroscientist 14(6):540-543.

Flynn G, Alexander D, Harris A, Whitford T, Wong W, Galletly C, Silverstein S, Gordon E, Williams LM. 2008. Increased absolute magnitude of gamma synchrony in first-episode psychosis. Schizophrenia research 105(1):262-271. 
Gschwandtner U, Zimmermann R, Pflueger MO, Riecher-Rössler A, Fuhr P. 2009. Negative symptoms in neuroleptic-naive patients with first-episode psychosis correlate with QEEG parameters. Schizophr Res 115(2-3):231-6.

Guerrero-Pedraza A, McKenna P, Gomar J, Sarro S, Salvador R, Amann B, Carrion M, Landin-Romero R, Blanch J, Pomarol-Clotet E. 2012. First-episode psychosis is characterized by failure of deactivation but not by hypo-or hyperfrontality. Psychological medicine 42(01):73-84.

Hirano Y, Oribe N, Kanba S, Onitsuka T, Nestor PG, Spencer KM. 2015. Spontaneous Gamma Activity in Schizophrenia. JAMA psychiatry.

Hof PR, Haroutunian V, Friedrich VL, Byne W, Buitron C, Perl DP, Davis KL. 2003. Loss and altered spatial distribution of oligodendrocytes in the superior frontal gyrus in schizophrenia. Biological psychiatry 53(12):1075-1085.

Holmes AP, Blair R, Watson G, Ford I. 1996. Nonparametric analysis of statistic images from functional mapping experiments. Journal of Cerebral Blood Flow \& Metabolism 16(1):7-22.

Holscher C, Schmid S, Pilz PK, Sansig G, van der Putten H, Plappert CF. 2005. Lack of the Metabotropic Glutamate Receptor Subtype 7 Selectively Modulates Theta Rhythm and Working Memory. Learning \& memory 12(5):450-455.

Hubl D, Koenig T, Strik WK, Garcia LM, Dierks T. 2007. Competition for neuronal resources: how hallucinations make themselves heard. The British Journal of Psychiatry 190(1):57-62.

Insel TR. 2010. Rethinking schizophrenia. Nature 468(7321):187-193.

Káradóttir R, Cavelier P, Bergersen LH, Attwell D. 2005. NMDA receptors are expressed in oligodendrocytes and activated in ischaemia. Nature 438(7071):1162-1166.

Kegeles LS, Mao X, Stanford AD, Girgis R, Ojeil N, Xu X, Gil R, Slifstein M, Abi-Dargham A, Lisanby SH. 2012. Elevated prefrontal cortex $y$-aminobutyric acid and glutamate-glutamine levels in schizophrenia measured in vivo with proton magnetic resonance spectroscopy. Archives of general psychiatry 69(5):449-459.

Kikuchi M, Koenig T, Wada Y, Higashima M, Koshino Y, Strik W, Dierks T. 2007. Native EEG and treatment effects in neuroleptic-naive schizophrenic patients: time and frequency domain approaches. Schizophrenia research 97(1):163-172.

Kim JH, Chien JH, Liu CC, Lenz FA. 2015. Painful cutaneous laser stimuli induce event-related gammaband activity in the lateral thalamus of humans. J Neurophysiol 113(5):1564-73.

Knyazev GG, Slobodskaya HR, Safronova MV, Sorokin OV, Goodman R, Wilson GD. 2003. Personality, psychopathology and brain oscillations. Personality and individual differences 35(6):1331-1349.

Knyazeva MG, Jalili M, Meuli R, Hasler M, De Feo O, Do KQ. 2008. Alpha rhythm and hypofrontality in schizophrenia. Acta psychiatrica Scandinavica 118(3):188-199.

Koch M, Schmiedt-Fehr C, Mathes B. 2015. Neuropharmacology of altered brain oscillations in schizophrenia. International Journal of Psychophysiology.

Kometer M, Schmidt A, Jäncke L, Vollenweider FX. 2013. Activation of serotonin 2A receptors underlies the psilocybin-induced effects on a oscillations, N170 visual-evoked potentials, and visual hallucinations. The Journal of Neuroscience 33(25):10544-10551.

Lee K-H, Williams LM, Breakspear M, Gordon E. 2003. Synchronous gamma activity: a review and contribution to an integrative neuroscience model of schizophrenia. Brain Research Reviews 41(1):57-78. 
Lukoff D, Nuechterlein K, Ventura J. 1986. Manual for the expanded brief psychiatric rating scale. Schizophr Bull 12:594-602.

Manchanda R, Malla A, Harricharan R, Cortese L, Takhar J. 2003. EEG abnormalities and outcome in firstepisode psychosis. Canadian journal of psychiatry. Revue canadienne de psychiatrie 48(11):7226.

Manchanda R, Norman R, Malla A, Harricharan R, Northcott S. 2008. EEG abnormalities and 3-year outcome in first episode psychosis. Acta psychiatrica Scandinavica 117(4):277-82.

Manchanda R, Norman R, Malla A, Harricharan R, Northcott S, Richard J. 2014. Electroencephalographic abnormalities and 5 -year outcome in first-episode psychosis. Canadian journal of psychiatry. Revue canadienne de psychiatrie 59(5):285-8.

Manchanda R, Norman R, Malla A, Harricharan R, Takhar J, Northcott S. 2005. EEG abnormalities and two year outcome in first episode psychosis. Acta psychiatrica Scandinavica 111(3):208-13.

Mazziotta J, Toga A, Evans A, Fox P, Lancaster J, Zilles K, Woods R, Paus T, Simpson G, Pike B. 2001. A probabilistic atlas and reference system for the human brain: International Consortium for Brain Mapping (ICBM). Philosophical Transactions of the Royal Society of London. Series B: Biological Sciences 356(1412):1293-1322.

Mesholam-Gately RI, Giuliano AJ, Goff KP, Faraone SV, Seidman LJ. 2009. Neurocognition in firstepisode schizophrenia: a meta-analytic review. Neuropsychology 23(3):315.

Michel CM, Murray MM, Lantz G, Gonzalez S, Spinelli L, Grave de Peralta R. 2004. EEG source imaging. Clinical neurophysiology : official journal of the International Federation of Clinical Neurophysiology 115(10):2195-222 .

Micheloyannis S, Pachou E, Stam CJ, Vourkas M, Erimaki S, Tsirka V. 2006. Using graph theoretical analysis of multi channel EEG to evaluate the neural efficiency hypothesis. Neuroscience letters 402(3):273-277.

Mu Y, Fan Y, Mao L, Han S. 2008. Event-related theta and alpha oscillations mediate empathy for pain. Brain research 1234:128-136.

Mulert C, Jäger L, Schmitt R, Bussfeld P, Pogarell O, Möller H-J, Juckel G, Hegerl U. 2004. Integration of fMRI and simultaneous EEG: towards a comprehensive understanding of localization and timecourse of brain activity in target detection. Neuroimage 22(1):83-94.

Nejad AB, Ebdrup BH, Siebner HR, Rasmussen H, Aggernæs B, Glenthøj BY, Baaré WF. 2011. Impaired temporoparietal deactivation with working memory load in antipsychotic-naive patients with firstepisode schizophrenia. World Journal of Biological Psychiatry 12(4):271-281.

Nunez PL, Srinivasan R. 2006. Electric fields of the brain: the neurophysics of EEG. Oxford university press.

Oertel-Knöchel V, Knöchel C, Rotarska-Jagiela A, Reinke B, Prvulovic D, Haenschel C, Hampel H, Linden DE. 2013. Association between psychotic symptoms and cortical thickness reduction across the schizophrenia spectrum. Cerebral Cortex 23(1):61-70.

Pascual-Marqui RD. 2007. Discrete, 3D distributed, linear imaging methods of electric neuronal activity. Part 1: exact, zero error localization. arXiv preprint arXiv:0710.3341.

Pascual-Marqui RD, Lehmann D, Koenig T, Kochi K, Merlo MC, Hell D, Koukkou M. 1999. Low resolution brain electromagnetic tomography (LORETA) functional imaging in acute, neuroleptic-naive, firstepisode, productive schizophrenia. Psychiatry Research: Neuroimaging 90(3):169-179. 
Pascual-Marqui RD, Lehmann D, Koukkou M, Kochi K, Anderer P, Saletu B, Tanaka H, Hirata K, John ER, Prichep L, Biscay-Lirio R, Kinoshita T. 2011. Assessing interactions in the brain with exact lowresolution electromagnetic tomography. Philos Trans A Math Phys Eng Sci 369(1952):3768-84.

Pascual-Marqui RD, Michel CM, Lehmann D. 1994. Low resolution electromagnetic tomography: a new method for localizing electrical activity in the brain. Int J Psychophysiol 18(1):49-65.

Pizzagalli D, Oakes T, Fox A, Chung M, Larson C, Abercrombie H, Schaefer S, Benca R, Davidson R. 2003. Functional but not structural subgenual prefrontal cortex abnormalities in melancholia. Molecular psychiatry 9(4):393-405.

Pol HEH, Schnack HG, Bertens MG, van Haren NE, van der Tweel I, Staal WG, Baaré WF, Kahn RS. 2014. Volume changes in gray matter in patients with schizophrenia.

Ramyead A, Kometer M, Studerus E, Koranyi S, Ittig S, Gschwandtner U, Fuhr P, Riecher-Rossler A. 2014. Aberrant Current Source-Density and Lagged Phase Synchronization of Neural Oscillations as Markers for Emerging Psychosis. Schizophr Bull.

Ramyead A, Studerus E, Kometer M, Uttinger M, Gschwandtner U, Fuhr P, Riecher-Rössler A. 2015. Prediction of psychosis using neural oscillations and machine learning in neuroleptic-naïve at-risk patients. World J Biol Psychiatry:1-11.

Riecher-Rössler A, Aston J, Ventura J, Merlo M, Borgwardt S, Gschwandtner U, Stieglitz RD. 2008. [The Basel Screening Instrument for Psychosis (BSIP): development, structure, reliability and validity]. Fortschr Neurol Psychiatr 76(4):207-16.

Riecher-Rössler A, Pflueger MO, Aston J, Borgwardt SJ, Brewer WJ, Gschwandtner U, Stieglitz RD. 2009. Efficacy of using cognitive status in predicting psychosis: a 7-year follow-up. Biol Psychiatry 66(11):1023-30.

Riecher-Rössler A, Gschwandtner U, Aston J, Borgwardt S, Drewe M, Fuhr P, Pflüger M, Radü W, Schindler C, Stieglitz RD. 2007. The Basel early-detection-of-psychosis (FEPSY)-study-design and preliminary results. Acta psychiatrica Scandinavica 115(2):114-125.

Roopun AK, Cunningham MO, Racca C, Alter K, Traub RD, Whittington MA. 2008. Region-specific changes in gamma and beta2 rhythms in NMDA receptor dysfunction models of schizophrenia. Schizophrenia bulletin 34(5):962-973.

Schmitt A, Hasan A, Gruber O, Falkai P. 2011. Schizophrenia as a disorder of disconnectivity. European archives of psychiatry and clinical neuroscience 261(2):150-154.

Schneider F, Habel U, Reske M, Kellermann T, Stöcker T, Shah NJ, Zilles K, Braus DF, Schmitt A, Schlösser R. 2007. Neural correlates of working memory dysfunction in first-episode schizophrenia patients: an fMRI multi-center study. Schizophrenia research 89(1):198-210.

Sumiyoshi T, Kawasaki Y, Suzuki M, Higuchi Y, Kurachi M. 2008. Neurocognitive assessment and pharmacotherapy towards prevention of schizophrenia: What can we learn from first episode psychosis. Clin Psychopharmacol Neurosci 6:57-64.

Tan H-Y, Choo W-C, Fones CS, Chee MW. 2005. fMRI study of maintenance and manipulation processes within working memory in first-episode schizophrenia. American Journal of Psychiatry 162(10):1849-1858.

Tenke CE, Kayser J, Manna CG, Fekri S, Kroppmann CJ, Schaller JD, Alschuler DM, Stewart JW, McGrath PJ, Bruder GE. 2011. Current source density measures of electroencephalographic alpha predict antidepressant treatment response. Biological psychiatry 70(4):388-94.

Théberge J, Bartha R, Drost DJ, Menon RS, Malla A, Takhar J, Neufeld RW, Rogers J, Pavlosky W, Schaefer B. 2002. Glutamate and glutamine measured with 4.0 T proton MRS in never-treated 
patients with schizophrenia and healthy volunteers. American Journal of Psychiatry 159(11):19441946.

Tsujimoto T, Shimazu H, Isomura Y. 2006. Direct recording of theta oscillations in primate prefrontal and anterior cingulate cortices. Journal of neurophysiology 95(5):2987-3000.

Uhlhaas PJ, Singer W. 2010. Abnormal neural oscillations and synchrony in schizophrenia. Nature reviews neuroscience 11(2):100-113.

Uhlhaas PJ, Singer W. 2013. High-frequency oscillations and the neurobiology of schizophrenia. Dialogues in clinical neuroscience 15(3):301.

Valkonen-Korhonen M, Purhonen M, Tarkka IM, Sipilä P, Partanen J, Karhu J, Lehtonen J. 2003. Altered auditory processing in acutely psychotic never-medicated first-episode patients. Cognitive brain research 17(3):747-758.

Velligan D, Prihoda T, Dennehy E, Biggs M, Shores-Wilson K, Crismon ML, Rush AJ, Miller A, Suppes T, Trivedi M. 2005. Brief psychiatric rating scale expanded version: How do new items affect factor structure? Psychiatry research 135(3):217-228.

Ventura J, Green MF, Shaner A, Liberman RP. 1993. Training and quality assurance with the Brief Psychiatric Rating Scale:" the drift busters.". International Journal of Methods in Psychiatric Research.

Wang R, Zhang Z, Qu J, Cao J. 2011. Phase synchronization motion and neural coding in dynamic transmission of neural information. Neural Networks, IEEE Transactions on 22(7):1097-1106.

Ward LM. 2003. Synchronous neural oscillations and cognitive processes. Trends in cognitive sciences 7(12):553-559.

Whitfield-Gabrieli S, Thermenos HW, Milanovic S, Tsuang MT, Faraone SV, McCarley RW, Shenton ME, Green Al, Nieto-Castanon A, LaViolette P. 2009. Hyperactivity and hyperconnectivity of the default network in schizophrenia and in first-degree relatives of persons with schizophrenia. Proceedings of the National Academy of Sciences 106(4):1279-1284.

Womelsdorf T, Johnston K, Vinck M, Everling S. 2010. Theta-activity in anterior cingulate cortex predicts task rules and their adjustments following errors. Proceedings of the National Academy of Sciences 107(11):5248-5253.

Woodward ND, Waldie B, Rogers B, Tibbo P, Seres P, Purdon SE. 2009. Abnormal prefrontal cortical activity and connectivity during response selection in first episode psychosis, chronic schizophrenia, and unaffected siblings of individuals with schizophrenia. Schizophrenia research 109(1):182-190.

Worrell GA, Lagerlund TD, Sharbrough FW, Brinkmann BH, Busacker NE, Cicora KM, O'Brien TJ. 2000. Localization of the epileptic focus by low-resolution electromagnetic tomography in patients with a lesion demonstrated by MRI. Brain Topography 12(4):273-282.

Yung AR, Phillips LJ, McGorry PD, McFarlane CA, Francey S, Harrigan S, Patton GC, Jackson HJ. 1998. Prediction of psychosis. A step towards indicated prevention of schizophrenia. Br J Psychiatry Suppl 172(33):14-20.

Zumsteg D, Lozano AM, Wennberg RA. 2006. Depth electrode recorded cerebral responses with deep brain stimulation of the anterior thalamus for epilepsy. Clinical Neurophysiology 117(7):1602-1609.

Zumsteg D, Wennberg R, Treyer V, Buck A, Wieser H. 2005. H215O or 13NH3 PET and electromagnetic tomography (LORETA) during partial status epilepticus. Neurology 65(10):1657-1660. 
Table 1: Demographic and clinical characteristics at EEG assessment

\begin{tabular}{|c|c|c|c|}
\hline & $\begin{array}{c}\mathrm{HC} \\
\mathrm{N}=29\end{array}$ & $\begin{array}{c}\text { FEP } \\
\mathrm{N}=31\end{array}$ & $p$ Value \\
\hline Gender: & & & 0.815 \\
\hline Women & $14(48.3 \%)$ & $13(41.9 \%)$ & \\
\hline Men & $15(51.7 \%)$ & $18(58.1 \%)$ & \\
\hline Age & $22.4(5.02)$ & $30.8(8.92)$ & $<0.05$ \\
\hline Years of education & $11.9(1.93)$ & $10.5(2.99)$ & $<0.05$ \\
\hline \multicolumn{4}{|l|}{ Antidepressants currently: } \\
\hline no & & 27 (87.1\%) & \\
\hline yes & & $4(12.9 \%)$ & \\
\hline \multicolumn{4}{|l|}{ Tranquilizer currently: } \\
\hline no & & $23(74.2 \%)$ & \\
\hline yes & & $8(25.8 \%)$ & \\
\hline BPRS Positive Symptoms & & $13.4(3.45)$ & \\
\hline BPRS Negative symptoms & & $5.79(2.36)$ & \\
\hline BPRS total score & & $55.0(12.1)$ & \\
\hline
\end{tabular}




\section{Figure Captions}

Figure 1: $\quad$ For illustrative purposes, the average current source density $(\mu \mathrm{A} / \mathrm{mm} 2)$ by group and frequency bands.

Figure 2: $\quad$ eLORETA statistical map of oscillatory differences in the (A) theta (B) alpha $1(\mathrm{C})$ beta2 and (D) gamma frequency bands between FEP and HC.

Figure 3: $\quad$ The lagged phase-synchronization of the (A) beta 1 and (B) beta 2 frequency band as a function of distance. Shaded areas cover regression coefficients with $\pm 1 \mathrm{SE}$.

Figure 4: $\quad$ (A) The lagged phase-synchronization of the beta 2 frequency bands as a function of distance for each of 4 different values of BPRS positive symptoms. (B) The lagged phase-synchronization of the beta 1 frequency band as a function of distance for each of 4 different values of BPRS negative symptoms. Shaded areas cover regression coefficients with $\pm 1 \mathrm{SE}$. 